\title{
Correspondence
}

\section{The unanticipated difficult airway}

To the Editor:

The article by Crosby $e t a l$ "The unanticipated difficult airway with recommendations for management"l and accompanying editorial "The difficult airway - a Canadian perspective" are important contributions. Four important points deserve additional commentary.

First, both articles considered and discussed the most useful and accurate definition of a difficult intubation and both politely indicated that the ASA definition of "more than three attempts" or "more than ten minutes" is lacking. I agree: a difficult intubation can be both time and number of attempts independent. ${ }^{3}$ The definition by Crosby of "I) more than two attempts with the same blade or; 2) a change in the blade or an adjunct to a direct laryngoscopy or; 3 ) use of an alternative device or technique following failed intubation with direct laryngoscopy" comes close to covering the clinical realities of "difficulty" for everyone. I would add "a Grade IIIB or Grade IV view". Grade III should be divided up into Grade IIIA = only proximal and distal half of epiglottis seen and Grade IIIB = only proximal half (tip) of epiglottis seen, because these views probably respond differently to therapeutic manoeuvres such as optimal external laryngeal manipulation and change in length and type of blade. ${ }^{3}$

Second, Finucane characterized an algorithm for the management of the unexpected difficult airway in the Crosby et al. article as a "simplified version of that presented in the ASA guideline." I want to emphasize that "simpler" should not mean "different" because intellectually and clinically both algorithms are the same.

Third, Finucane and Crosby emphasize the importance of teaching airway management skills and I am as puzzled as they by the fact that the majority of teaching programs in North America do not have a structured teaching program in this area. The only explanation I have for such an illogical situation is that most older anesthesiologists come from an era when they and others thought that all anesthesiologists were the 'airway experts', so why would 'airway experts' need more or different training? The ASA Closed Claim. Study showed anesthesiologists were not 'expert' enough to prevent complications due to management of the airway from being the most frequent and severe of all complications related to the administration of anesthesia. Our program at UCSD in patients has worked well but the supervising faculty must exercise tight control, good common sense and judgement. ${ }^{4}$ We use mannequins only in the beginning of a formal airway rotation to help the resident get off to a better start in patients. Thus, I do not feel informed consent is necessary because the anesthesia and airway care that is administered is as good as or better, from both trauma and success points of view, as they might otherwise receive. ${ }^{5}$

Finally, I am puzzled by Dr. Finucane's characterization of Crosby's article as "displaying a distinctively Canadian viewpoint". I do not argue that such a thing exists but what is it? I was very relieved to learn that important local / territorial differences do not exist because it is hard to imagine that such a fundamentally important life-taking/life-giving problem as the management of the difficult airway would have fundamentally different answers.

Jonathan L. Benumof MD

San Diego, California, USA

\section{REFERENCES}

1 Crosby ET, Cooper RM, Douglas MJ, et al. The unanticipated difficult airway with recommendations for management. Can J Anaesth 1998; 45: 757-76.

2 Finucane B. The difficult airway - a Canadian perspective (Editorial). Can J Anaesth 1998; 45: 713-8.

3 Benumof $J$. Difficult laryngoscopy: obtaining the best view (Editorial). Can J Anaesth 1994; 41: 361-5.

4 Cooper SD, Benumof $J$. Teaching management of the airway: The UCSD airway rotation. In: Benumof JL (Ed.). Airway Management: Principles and Practice. Philadelphia, PA: Mosby CV, 1996: 903-10.

5 Benumof $J L$, Cooper $S D$. Teaching airway management skills: what about patient consent? Anaesthesia 1996; 85: 438-9.

\section{REPLY:}

I would like to thank Dr. Benumof for his interest and thoughtful commentary. I think that we should teach what we practice. We rely, in many instances, on technology and techniques that we know are dated and less than optimum for the task at hand. Rose reported that difficult laryngoscopy was most often managed with persistent attempts at direct laryngoscopy and that the use of alternative approaches to tracheal intubation was uncommon. I In a recent survey, Rosenblatt presented 
difficult airway scenarios involving co-operative adult patients who required tracheal intubation; physicians were asked to identify their preferred management technique. Experienced practitioners tended to use higher risk induction techniques. The use of adjuncts to the direct laryngoscope or alternative devices including the Bullard laryngoscope or the lighted stylet was uncommon,. In the patient with a bistory of previous difficult intubation, $60 \%$ of experienced practitioners would induce general anesthesia and 59\% would proceed with direct laryngoscopy. In balf of the institutions surveyed, the lighted stylet, retrograde intubation kits, the Combitube, gum elastic bougies, or the Bullard laryngoscope were unavailable and $17 \%$ of anesthesiologists did not have access to the laryngeal mask airway. ${ }^{2}$

It is likely that the major reason for the lack of motivation within the specialty to learn, apply and teach newer techniques in airway management, is that airway disasters are sufficiently rare that one may function for a pery long time under the illusion that it will never bappen. Mannequins and simulators will likely have their greatest utility for training with techniques that may constitute a small but finite and different risk to patients (i.e. esophageal injury after Combitube insertion).

Edward Crosby MD

Ottawa, Ontario

\section{REFERENCES}

1 Rose $D K$, Coben $M M$. The airway: problems and predictions in 18,500 patients. Can J Anaesth 1994; 41: 372-83.

2 Rosenblatt WH, Wagner PJ, Ovassapian A, Kain ZN. Practice patterns in managing the difficult airway by anesthesiologists in the United States. Anesth Analg 1998; 87: 153-7.

\section{REPLY:}

We are pleased to receive endorsement of our deliberations on the unanticipated difficult airway from Benumof who is a keen and learned master of this important topic.

Benumof suggests further modification of the definition by adding a fourth category. However, he already stated that Crosby et al.'s definition "comes pretty close to objectively covering the clinical realities of difficulty for everyone". Whatever definition we agree upon, we must strive for universal agreement so that we can collect some meaningful data on this important topic world-wide. Benumof was surprised that Crosby et al. did not comment on the algorithm in the text of the article. I suggest that the Crosby et al. algorithm is a simplified version of the ASA algorithm.
As both the Crosby and ASA algorithms are comparable most clinicians would opt for a more concise persion. One of the purposes of an algorithm is to encourage clear thinking. Complicated road maps are distracting and sometimes lead people astray.

Finally, Benumof is puzzled once again by my observation that Crosby et al.'s paper expresses a "distinctly Canadian viewpoint". I agree that the content in Crosby et al.'s article does not differ very much from the American viewpoint. It really boils down to a difference in style.

Brendan T. Finucane MBBCH FRCA FRCPC

Edmonton, Alberta

\section{REFERENCE}

1 Crosby ET, Cooper RM, Douglas MJ, et al. The unanticipated difficult airway with recommendations for management. Can J Anaesth 1998; 45: 757-76.

\section{$L M A / F O I$ and $C A F G$}

To the Editor:

We disagree with the CAFG statement that fibreoptic intubation is "compromised in the .... anesthetized patient." They cite but one review article in support, based on the author's "personal experience supplemented by a limited literature review". 2 This, despite the citation methodology that claimed: "only titles including original data were included; excluded were ... titles with no original data (reviews, editorials, comments"). The CAFG has ignored the considerable evidence-based literature on fibreoptic intubation of anesthetized patients.

We have demonstrated that junior residents, using anesthetized patients, learn fibreoptic as readily as laryngoscopic intubation, without increasing morbidity or surgical delay. ${ }^{3}$ Others have reported similar results. ${ }^{4-8}$ Although we do not advocate this technique for patients with recognized difficult airways or airway pathology, we have found expertise gained during elective fibreoptic intubation of anesthetized patients translates into equal facility in fibreoptic.intubation in unrecognized difficult laryngoscopy in anesthetized patients, and also in awake fibreoptic intubation, (as did Marsch. ${ }^{9}$ )

Interestingly, the CAFG displays an open attitude to newer devices - the lighted stylette and intubating laryngeal mask airway. Could the authors been seduced by new, and therefore glamorous, equipment? New devices are appealing - all is yet to be explored, yet to be written. Career investigators might find such 